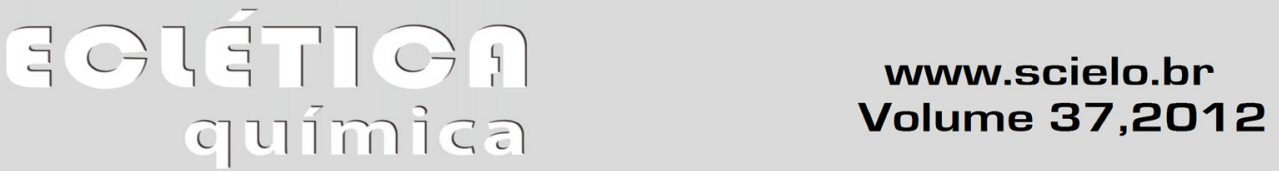

\title{
MULTI-ELEMENT DETERMINATION OF CALCIUM, POTASSIUM AND MAGNESIUM IN MEDICINAL PLANT BY HIGH-RESOLUTION CONTIN- UUM SOURCE ATOMIC ABSORPTION SPECTROMETRY
}

\author{
Tiago Varão Silva, Jardes Figuerêdo Rêgo, Mercedes de Moraes, \\ Alberto José Cavalheiro, José Anchieta Gomes Neto ${ }^{1}$ \\ Instituto de Química, Universidade Estadual Paulista, CP 355, 14801-970 Araraquara - SP, Brasil \\ Jorge Luiz Raposo Júnior \\ Faculdade de Ciências Exatas e Tecnologia, Universidade Federal da Grande Dourados, CP 533, 79804-970 Dourados - MS, Brasil
}

1 Autor para correspondência. Tel: 16 33019611; Fax: 1633019692.

E-mail: anchieta@iq.unesp.br(J.A. Gomes Neto)

A simple and rugged method to determine $\mathrm{Ca}, \mathrm{K}$ and $\mathrm{Mg}$ in a single aliquot of medicinal plants by high-resolution continuum source flame atomic absorption spectrometry is proposed. Secondary lines for Ca $(239.856 \mathrm{~nm})$ and $\mathrm{K}$ $(404.414 \mathrm{~nm})$, and the alternate line measured at wing of the secondary line for $\mathrm{Mg}$ at $202.588 \mathrm{~nm}$ allowed calibration within the $20-500 \mathrm{mg} \mathrm{L}^{-1} \mathrm{Ca}$ and $\mathrm{K}$, and $1.0-80 \mathrm{mg} \mathrm{L}^{-1} \mathrm{Mg}$. Twenty samples and three plant certified materials were analyzed. Results were in agreement at a $95 \%$ confidence level with reference values. Limits of detection were $2.4 \mathrm{mg} \mathrm{L}^{-1} \mathrm{Ca}, 1.9 \mathrm{mg} \mathrm{L}^{-1} \mathrm{~K}_{\text {and }} 0.3 \mathrm{mg} \mathrm{L}^{-1} \mathrm{Mg}$. The $\mathrm{RSD}(\mathrm{n}=12)$ were $\leq 5.1 \%$ and recoveries were between 83 and $108 \%$ for all analytes.

Keywords: macronutrients; medicinal plants; HR-CS FAAS

\section{INTRODUÇÃO}

Conhecer a composição de plantas medicinais é uma forma de garantir a população brasileira o acesso seguro e o uso racional dessas plantas, promover o uso sustentável da biodiversidade e desenvolver a cadeia produtiva nacional. As plantas medicinais podem apresentar alto valor nutritivo e a determinação do teor total de um elemento é relevante [1]. O estabelecimento de métodos de análise desta classe de plantas voltados a um controle de qualidade eficiente, visando minimizar a probabilidade de ocorrência de problemas toxicológicos, afigura-se como promissor. Entre as técnicas analíticas, a espectrometria de absorção atômica [2] ocupa um espaço importante no campo das análises químicas elementares.

A determinação de $\mathrm{Ca}, \mathrm{K}$ e $\mathrm{Mg}$ em plantas medicinais por espectrometria de absorção atômica em chama é feita frequentemente em espectrômetros com fontes de radiação discretas (LS FAAS) [3]. Esta determinação é uma tarefa simples, contudo trabalhosa e morosa porque requer otimizações instrumental e operacional específicas para cada elemento, tais como: a troca e o condicionamento das fontes de radiação, os espaços temporais relativamente longos entre calibrações, os ajustes da diluição da amostra aos respectivos intervalos lineares das curvas analíticas que consomem tempo e aumentam os custos analíticos. Estes aspectos negativos podem ser minimizados se vários elementos puderem ser determinados conjuntamente numa única alíquota da amostra [4]. Apesar dos teores de Mg em tecidos vegetais serem inferiores aos usualmente encontrados para Ca e K [5], a utilização da linha atômica principal ou secundária do Mg não permite determiná-lo na mesma alíquota de amostra utilizada para determinar $\mathrm{Ca}$ e $\mathrm{K}$, uma vez que os limites superiores de resposta linear são baixos (0,5 e $10 \mathrm{mg} \mathrm{L}^{-1} \mathrm{Mg}$, respectivamente), exig- 
indo diluições subsequentes da amostra.

Diferentemente da LS AAS, a espectrometria de absorção atômica em chama com fonte contínua e alta resolução (HR-CS FAAS) [6] possibilita monitorar a absorbância dos elementos em diferentes comprimentos de onda, desde os mais sensíveis até aqueles localizados nas regiões adjacentes ao máximo de uma linha (side pixel registration - SPR) [7], viabilizando assim determinações multielementares em ampla faixa de concentração numa mesma alíquota de amostra [8].

Este trabalho consiste em avaliar a redução da sensibilidade e o aumento do intervalo linear de trabalho com vistas ao desenvolvimento de um método simples e rápido para determinar de modo sequencial rápido $\mathrm{Ca}$, $\mathrm{K}$ e Mg numa mesma alíquota de digeridos de plantas medicinais por HR-CS FAAS.

\section{PARTE EXPERIMENTAL}

\section{Reagentes, soluções analíticas e amostras}

Todas as soluções foram preparadas em água deionizada de alta pureza obtida a partir de um sistema de osmose reversa Millipore Rios $5^{\mathrm{TM}}$ combinado com deionizador Millipore Milli-Q academic. Para a digestão das amostras de folhas de plantas medicinais, acido nítrico $70 \%$ (Spectrum Chemical $^{\circledR}$ ) e peróxido de hidrogênio $30 \%(\mathrm{~m} / \mathrm{v})$ de grau analítico foram utilizados.

Solução estoque $1000 \mathrm{mg} \mathrm{L}^{-1} \mathrm{Mg}$ foi preparada a partir do respectivo padrão espectroscópico $\left(\right.$ Chemis $\left.^{\circledR}\right)$ por diluição do conteúdo da ampola em balão volumétrico de $1000 \mathrm{~mL}$ com água.

Solução estoque $5009 \mathrm{mg} \mathrm{L}^{-1} \mathrm{Ca}$ foi preparada a partir de $\mathrm{CaCO}_{3}$ (Mallinckrodt Baker, Xalostoc, México). Uma massa de 3,127 $\mathrm{g} \mathrm{CaCO}_{3}$ previamente seco em estufa $\left(100^{\circ} \mathrm{C}\right)$ foi dispersa em cerca de $100 \mathrm{~mL}$ de água e dissolvida após adição de pequenas alíquotas de $\mathrm{HNO}_{3}$ concentrado. A solução resultante foi diluída a $250 \mathrm{~mL}$ com água deionizada e em seguida padronizada com EDTA.

Solução estoque $5000 \mathrm{mg} \mathrm{L}^{-1} \mathrm{~K}$ foi preparada a partir da dissolução de 2,383 $\mathrm{g} \mathrm{KCl}$ (Mallinckrodt Baker, Xalostoc, México) previamente seco em estufa $\left(100{ }^{\circ} \mathrm{C}\right)$, e diluição a $250 \mathrm{~mL}$ com água deionizada.

Solução estoque $5 \%(\mathrm{~m} / \mathrm{v})$ La foi preparada pela dissolução de 58,6 $\mathrm{g} \mathrm{La}_{2} \mathrm{O}_{3}$ (J. T. Baker®, México) em $250 \mathrm{~mL}$ de ácido clorídrico concentrado e diluição a $1000 \mathrm{~mL}$ com água. Todas as soluções es- toque foram armazenadas em frascos de polipropileno (Nalgene ${ }^{\circledR}$, EUA) de alta densidade e mantidas sob refrigeração.

Para a determinação dos teores de $\mathrm{Ca}$, $\mathrm{Mg}$ e K, soluções analíticas multielementares contendo 20 - $500 \mathrm{mg} \mathrm{L}^{-1} \mathrm{Ca}$, K e 1,0 - $80 \mathrm{mg} \mathrm{L}^{-1} \mathrm{Mg}$ foram preparadas por diluição apropriada de soluções estoque individuais em meio 1,0\% (v/v) $\mathrm{HNO}_{3}$ e 1,0\% (m/v) La. As amostras de plantas medicinais foram adquiridas na Casa de Ervas São Francisco, Araraquara-SP, ou junto ao Centro Pluridisciplinar de Pesquisas Químicas, Biológicas e Agrícolas - CPQBA/Unicamp.

Materiais de referência certificados 1572 Citrus Leaves, 1515 Apple Leaves e 1547 Peach Leaves do National Institute of Standards and Technology (Gaithersburg, EUA) foram utilizados para avaliar a exatidão do método. As soluções analíticas e amostras foram introduzidas no sistema nebulizador/queimador do espectrômetro com auxílio do amostrador SFS6. Todas as medidas foram feitas em triplicata. A vidraria e os frascos empregados no preparo e armazenamento das soluções foram previamente limpos por imersão em banho de $\mathrm{HNO}_{3} 10 \%(\mathrm{v} / \mathrm{v})$ por $24 \mathrm{~h}$ e posteriormente lavados com água deionizada.

\section{Instrumentação e acessórios}

A determinação dos elementos $\mathrm{Ca}, \mathrm{K}$ e Mg foi feita com auxílio de um espectrômetro de absorção atômica em chama com fonte contínua e de alta resolução ContrAA 300 (Analytik Jena ${ }^{\circledR}$, Alemanha) equipado com uma lâmpada de arco curto de xenônio 300W (GLE, Berlim, Alemanha) com fonte de radiação contínua compreendida entre 190 a $900 \mathrm{~nm}$ e queimador de $50 \mathrm{~mm}$. Devido aos elevados teores naturais desses macronutrientes em plantas medicinais, as medidas de absorbância foram feitas nos comprimentos de onda secundários do $\mathrm{Ca}(239,856 \mathrm{~nm})$ e do K (404,414 nm). As absorbâncias do Mg foram medidas no comprimento de onda adjacente $(202,588 \mathrm{~nm})$ ao secundário $(202,582 \mathrm{~nm})$, ou seja, localizado em parte da asa do sinal de absorção atômica do elemento. Este procedimento permitiu estender a faixa linear de trabalho para $\mathrm{Mg}$ e determinar conjuntamente $\mathrm{Ca}, \mathrm{K}$ e Mg na mesma alíquota de digerido da amostra. No caso do procedimento de medida por SPR, os comprimentos de onda $202,575 \mathrm{~nm}, 202,577 \mathrm{~nm}, 202,586 \mathrm{~nm}$ e 202,588 $\mathrm{nm}$ foram avaliados. Como o objetivo era re- 
duzir a sensibilidade da medida para $\mathrm{Mg}$, a absorbância integrada no comprimento de onda foi equivalente a 1 pixel, a mínima permitida pelo programa gerenciador do espectrômetro.

A mistura ar-acetileno (acetileno 99,7\%, Air Liquide, Sertãozinho-SP, Brasil) foi utilizada para atomizar $\mathrm{Ca}, \mathrm{K}, \mathrm{Mg}$. A taxa de aspiração da amostra foi fixada em 5,0 $\mathrm{mL} \mathrm{min}^{-1}$, e os demais parâmetros instrumentais (vazão dos gases, altura de observação e tipo de queimador) foram otimizados e constam da Tabela 1 .

Tabela 1. Parâmetros instrumentais otimizados para determinar $\mathrm{Ca}, \mathrm{K}$ e Mg em plantas medicinais por HR-CS FAAS.

\begin{tabular}{ccccc}
\hline $\begin{array}{c}\text { Elemento - linha } \\
\text { atômica }(\mathrm{nm})\end{array}$ & $\begin{array}{c}\text { Altura de } \\
\text { observação }(\mathrm{mm})\end{array}$ & $\begin{array}{c}\text { Acetileno } \\
\left(\mathrm{L} \mathrm{h}^{-1}\right)\end{array}$ & $\begin{array}{c}\text { ar } \\
\left(\mathrm{L} \mathrm{h}^{-1}\right)\end{array}$ & $\begin{array}{c}\text { Tipo de } \\
\text { queimador }(\mathrm{mm})\end{array}$ \\
\hline $\mathrm{Ca}-239,856$ & 7 & 70 & 514 & 50 \\
$\mathrm{~K}-404,414$ & 6 & 60 & 514 & 50 \\
$\mathrm{Mg}-202,588$ & 7 & 70 & 514 & 50 \\
\hline
\end{tabular}

As amostras de plantas medicinais foram secas em estufa com circulação forçada de ar (TE-394/2 Tecnal $\left.^{\circledR}\right)$ a $40^{\circ} \mathrm{C}$ por $72 \mathrm{~h}$ e moídas em moinho de facas (Willey Tecnal ${ }^{\circledR} 648$ ) com peneira de abertura de aproximadamente $0,84 \mathrm{~mm}$ (20 mesh). A digestão das amostras foi feita em forno de microondas Anton Paar Multiwave ${ }^{\circledR} 300$ (Graz, Áustria) de 48 posições equipado com frascos de polietileno com capacidade de $25 \mathrm{~mL}$.

Um espectrômetro de absorção atômica em chama e com fonte de linhas Perkin-Elmer AAnalyst $^{\circledR} 100$ foi empregado para comparação de resultados.

\section{croondas}

Decomposição ácida assistida por radiação mi-

O preparo de soluções das amostras de plantas medicinais e dos materiais de referência utilizados na avaliação do método de determinação de $\mathrm{Ca}, \mathrm{K}$, e $\mathrm{Mg}$ foi feito em forno de microondas com controle de pressão e temperatura [9]. Massas de aproximadamente $0,25 \mathrm{~g}$ de folhas secas e moídas das plantas medicinais Melissa (Melissa officinalis ), Ginkgo Biloba (Ginkgo biloba L.), Hortelã (Mentha s.p.), Sene (Senna alexandrina), Cáscara Sagrada (Rhamnus purshiana), Graviola (Annona muricata), Poejo (Mentha longifólia), Ginseng (Panax ginseng), Pata de Vaca (Bauhinia forficata), Erva Doce (Pimpinella anisum), Boldo do
Chile (Plectranthus barbatus Andrews), Camomila (Matricaria chamomilla), Centelha Asiática (Centella asiatica), Guaçatonga (Casearia sylvestris), Chapéu de Couro (Echinodorus grandiflorus), Erva Cidreira (Lippia alba), Espinhadeira Santa (Maytenus ilicifolia), Guaco (Mikania glomerata), Carqueja (Baccharis crispa Spreng) e Cavalinha (Equisetum arvense), foram pesadas com precisão de $0,1 \mathrm{mg}$, transferidas para os frascos digestores do forno de microondas seguidas da adição de alíquotas de 3,0 $\mathrm{mL}$ de $\mathrm{HNO}_{3}+1,0 \mathrm{~mL}$ de $\mathrm{H}_{2} \mathrm{O}_{2}+2,0 \mathrm{~mL}$ de água. Em seguida, os frascos contendo amostra e reagentes foram dispostos no interior das "jaquetas" do forno de microondas, acomodados no rotor e então submetidos ao programa de aquecimento proposto para decomposição dos tecidos vegetais [10]. Os digeridos foram transferidos para balões volumétricos de $25 \mathrm{~mL}$ e o volume completado com água deionizada em meio de 1,0\% (m/v) La. Todas as soluções de amostras foram armazenadas em frascos de polipropileno de alta densidade (Nalgene, Rochester, EUA) e mantidas sob refrigeração até o momento da análise.

\section{RESULTADOS E DISCUSSÃO}

Vários experimentos foram realizados visando estabelecer o intervalo linear das curvas analíticas e os comprimentos de onda ótimos para a determinação dos três elementos num mesmo digerido. Considerando que os analitos em questão são macronutrientes [11], as medidas foram realizadas utilizando-se apenas 1 pixel, em cada comprimento de onda empregado. Inicialmente foram realizadas medidas nos comprimentos de onda mais sensíveis $(422,673 \mathrm{~nm}-\mathrm{Ca}, 766,490 \mathrm{~nm}$ $\mathrm{Ke} 285,213 \mathrm{~nm}$ - Mg) que forneceram os seguintes intervalos lineares: $0,1 \mathrm{a} 10 \mathrm{mg} \mathrm{L}^{-1} \mathrm{Ca}, 0,1 \mathrm{a} 10 \mathrm{mg} \mathrm{L}^{-1} \mathrm{~K}$ e 0,1 a 4,0 $\mathrm{mg} \mathrm{L}^{-1} \mathrm{Mg}$. Esses intervalos não atendiam a faixa de aplicação em virtude das altas concentrações de $\mathrm{Ca}$, $\mathrm{K}$ e $\mathrm{Mg}$ nos digeridos das amostras. Optou-se então por construir curvas analíticas utilizando os comprimentos de ondas secundários dos elementos: 239,856 nm (Ca), $404,414 \mathrm{~nm}(\mathrm{~K})$ e $202,582 \mathrm{~nm}(\mathrm{Mg})$. Nesta situação, as faixas lineares obtidas foram de 10 a $250 \mathrm{mg} \mathrm{L}^{-1} \mathrm{Ca} ; 10$ a $250 \mathrm{mg} \mathrm{L}^{-1} \mathrm{~K}$ e 0,5 a $5 \mathrm{mg} \mathrm{L}^{-1} \mathrm{Mg}$. Visando diminuir ainda mais a sensibilidade, estes mesmos experimentos foram repetidos empregando-se o queimador de $50 \mathrm{~mm}$, em vez daquele de $100 \mathrm{~mm}$ utilizado nos experimentos anteriores. Nestas condições, os intervalos lineares obtidos foram 20 a $500 \mathrm{mg} \mathrm{L}^{-1} \mathrm{Ca}, 20$ a $500 \mathrm{mg} \mathrm{L}^{-1} \mathrm{~K}$ e 1,0 a $10 \mathrm{mg} \mathrm{L}^{-1} \mathrm{Mg}$. Os valores de absorbâncias de Ca e K obtidos para a grande maioria dos digeridos das amostras 
situaram-se entre os limites inferiores e superiores destas curvas. Por outro lado, várias amostras apresentaram teores de $\mathrm{Mg}$ superiores a $10 \mathrm{mg} \mathrm{L}^{-1}$ (limite superior de resposta linear), exigindo diluições sucessivas. Em seguida avaliou-se a influência do SPR na absorbância do $\mathrm{Mg}$. Os comprimentos de onda 202,575 nm, 202,577 $\mathrm{nm}, 202,586 \mathrm{~nm}$ e $202,588 \mathrm{~nm}$, localizados na asa da linha secundária do $\mathrm{Mg}$, forneceram respectivamente os seguintes intervalos lineares $\left(\mathrm{em} \mathrm{mg} \mathrm{L}^{-1}\right): 1,0-30 ; 1,0$ $20 ; 1,0-35 ; 1,0-80$. A linha 202,588 nm foi escolhida para os experimentos subsequentes porque forneceu o mais amplo intervalo de trabalho e o maior limite superior de resposta linear. Desta maneira, os comprimentos de onda ideais para determinar conjuntamente esses três elementos em alíquotas de digeridos de plantas medicinais sem a necessidade de diluição corresponde aos comprimentos de onda $239,856 \mathrm{~nm}$ para $\mathrm{Ca}, 404,414$ $\mathrm{nm}$ para $\mathrm{K}$ e 202,588 $\mathrm{nm}$ para $\mathrm{Mg}$. As curvas analíticas otimizadas para determinação $\mathrm{Ca}, \mathrm{K}$ e $\mathrm{Mg}$ num mesmo digerido, foram construídas e estão ilustradas na Figura 1.
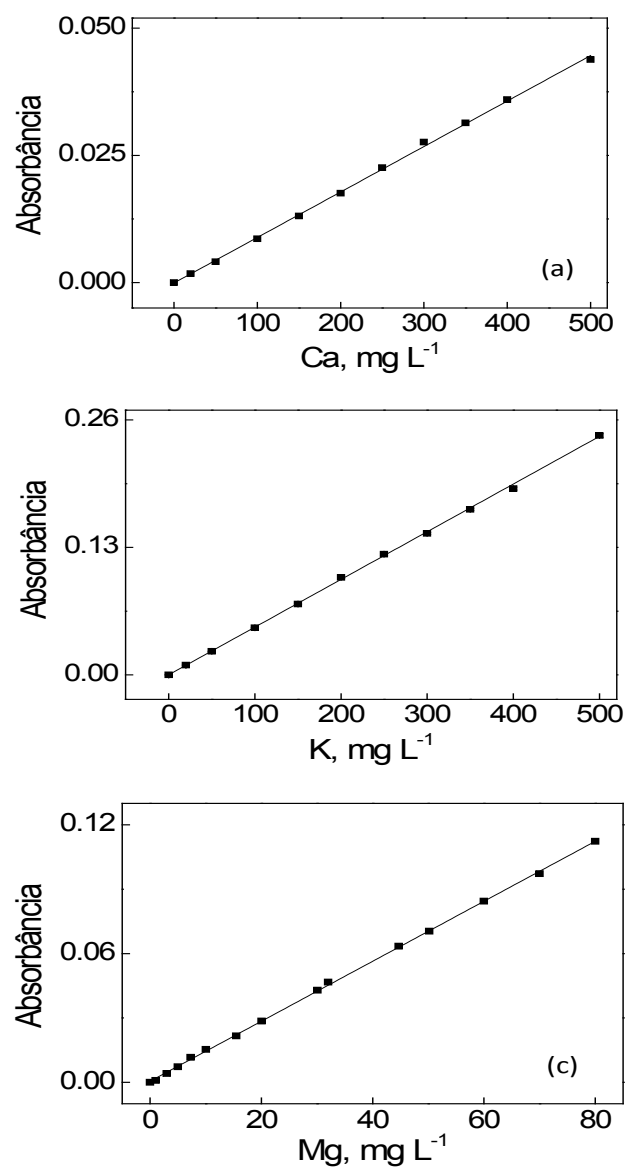

Figura 1. Curvas analíticas otimizadas para (a) Ca em 239,856 nm ( $\lambda$ secundário); (b) $\mathrm{K}$ em 404,414 nm ( $\lambda$ secundário); (c) $\mathrm{Mg}$ em $202,588 \mathrm{~nm}$ (asa do $\lambda$ secundário), obtidas por HR-CS FAAS com absorbância integrada no comprimento de onda equivalente a 1 pixel, empregando-se queimador de $50 \mathrm{~mm}$.
Nessas condições, os limites de quantificação [12] obtidos por HR-CS FAAS foram $8,0 \mathrm{mg} \mathrm{L}^{-1} \mathrm{Ca}$, $6,3 \mathrm{mg} \mathrm{L}^{-1} \mathrm{~K}$ e 1,0 $\mathrm{mg} \mathrm{L}^{-1} \mathrm{Mg}$. A precisão associada a 12 medidas sucessivas de uma solução contendo $100 \mathrm{mg}$ $\mathrm{L}^{-1} \mathrm{Ca}, 200 \mathrm{mg} \mathrm{L}^{-1} \mathrm{Ke} 50 \mathrm{mg} \mathrm{L}^{-1} \mathrm{Mg}$ foi de 4,3\%, 3,9\% e $5,1 \%$, respectivamente. As linhas 239,856 nm, 404,414 $\mathrm{nm}$ e 202,588 $\mathrm{nm}$ permitiram determinar respectivamente $\mathrm{Ca}, \mathrm{K}$ e $\mathrm{Mg}$ em todas as amostras de plantas sem diluição de seus digeridos. As principais figuras de mérito analítico dessas linhas utilizadas para calibrar o espectrômetro na determinação de $\mathrm{Ca}, \mathrm{K}$ e $\mathrm{Mg}$ estão resumidas na Tabela 2.

Tabela 2. Figuras de mérito analítico das linhas selecionadas para análise.

\begin{tabular}{cccccccc}
\hline Elemento & $\begin{array}{c}\text { Linha Atômica } \\
(\mathrm{nm})\end{array}$ & $\begin{array}{c}\text { Intervalo } \\
\text { Linear } \\
\left(\mathrm{mg} \mathrm{L}^{-1}\right)\end{array}$ & $\begin{array}{c}\text { Sensibilidade } \\
\left(\mathrm{A} \mathrm{L} \mathrm{mg}^{-1}\right)\end{array}$ & $\mathrm{R}$ & $\begin{array}{c}\text { LOD } \\
\left(\mathrm{mg} \mathrm{L}^{-1}\right)\end{array}$ & $\begin{array}{c}\text { LOQ } \\
\left(\mathrm{mg} \mathrm{L}^{-1}\right)\end{array}$ & $\begin{array}{c}\text { R.S.D } \\
(\%)\end{array}$ \\
\hline $\mathrm{Ca}$ & 239,856 & $20-500$ & $8,9.10^{-5}$ & 0,9991 & 2,4 & 8,0 & 4,3 \\
$\mathrm{~K}$ & 404,414 & $20-500$ & $4,8.10^{-4}$ & 0,9995 & 1,9 & 6,3 & 3,9 \\
$\mathrm{Mg}$ & 202,588 & $1,0-80$ & $1,4.10^{-3}$ & 0,9977 & 0,3 & 1,0 & 5,1 \\
\hline
\end{tabular}

Tabela 3. Resultados (média \pm desvio padrão) expressos em mg g-1 da determinação $(n=3)$ de $\mathrm{Ca}, \mathrm{K}$ e $\mathrm{Mg}$ nos materiais de referência certificados 1572 Citrus Leaves, 1515 Apple Leaves e 1547 Peach Leaves por HR-CS FAAS

\begin{tabular}{ccccccc}
\hline \multirow{2}{*}{ Analito } & \multicolumn{2}{c}{ Citrus Leaves } & \multicolumn{2}{c}{ Apple Leaves } & \multicolumn{2}{c}{ Peach Leaves } \\
\cline { 2 - 7 } & obtido & certificado & obtido & certificado & obtido & certificado \\
\hline $\mathrm{Ca}$ & $3,06 \pm 0,21$ & $3,15 \pm 0,10$ & $1,53 \pm 0,01$ & $1,53 \pm 0,02$ & $1,51 \pm 0,06$ & $1,56 \pm 0,02$ \\
$\mathrm{~K}$ & $1,79 \pm 0,04$ & $1,82 \pm 0,06$ & $1,58 \pm 0,06$ & $1,61 \pm 0,02$ & $2,37 \pm 0,08$ & $2,43 \pm 0,03$ \\
$\mathrm{Mg}$ & $0,52 \pm 0,03$ & $0,58 \pm 0,03$ & $0,26 \pm 0,01$ & $0,27 \pm 0,01$ & $0,42 \pm 0,01$ & $0,43 \pm 0,01$ \\
\hline
\end{tabular}

A exatidão do método proposto foi avaliada após análise de três materiais de referência e os resultados obtidos para $\mathrm{Ca}, \mathrm{K}$ e $\mathrm{Mg}$ não foram significativamente diferentes dos valores certificados ao nível de 95\% de confiança (Tabela 3 ).

Após análise dos materiais de referência certificados e avaliação positiva da eficiência do método para determinar $\mathrm{Ca}, \mathrm{K}$ e $\mathrm{Mg}$ em material vegetal (plantas medicinais), o método foi em seguida aplicado na determinação conjunta desses três analitos em 20 amostras de plantas medicinais amplamente comercializadas e consumidas no país (Tabela 4).

As concentrações dos analitos nos digeridos das amostras de plantas medicinais analisadas variaram de 25,86 - 475,2 $\mathrm{mg} \mathrm{L}^{-1} \mathrm{Ca}, 36,88$ - 402,5 $\mathrm{mg} \mathrm{L}^{-1} \mathrm{~K}$ e 3,14 - 80,88 $\mathrm{mg} \mathrm{L}^{-1} \mathrm{Mg}$. Essas concentrações estão situ- 
adas dentro das respectivas faixas lineares de trabalho definidas a partir das calibrações obtidas para as linhas secundárias do Ca $(239,856 \mathrm{~nm})$ e K $(404,414 \mathrm{~nm})$ e a linha alternativa do $\mathrm{Mg}(202,588 \mathrm{~nm})$, revelando que o método desenvolvido atendeu os objetivos propostos. Os teores dos analitos nas amostras de plantas medicinais analisadas expressos em $\mathrm{mg} \mathrm{g}^{-1}$ (massa seca) estão compilados na Tabela 4. Os resultados apresentados pelo método proposto não foram significativamente diferentes daqueles obtidos com a técnica alternativa (LS FAAS) ao nível de 95\% de confiança. Testes de adição e recuperação de analito conduzidos em cinco digeridos de amostras forneceram os seguintes intervalos de recuperação: 88 - 104\% (Ca), 89 - 105\% (K) e 83 - 108\% $(\mathrm{Mg})$.

Tabela 4. Teores de $\mathrm{Ca}, \mathrm{K}$ e Mg (em mg g-1, média \pm desvio padrão) determinados $(\mathrm{n}=3)$ em plantas medicinais empregando o método proposto por espectrometria de absorção atômica com fonte contínua e alta resolução (CS AAS) e por espectrometria de absorção atômica com fonte de linhas (LS AAS)

\begin{tabular}{|c|c|c|c|c|c|c|}
\hline \multirow{2}{*}{ Amostra } & \multicolumn{2}{|c|}{$\mathrm{Ca}$} & \multicolumn{2}{|c|}{$\mathrm{K}$} & \multicolumn{2}{|c|}{$\mathrm{Mg}$} \\
\hline & CS AAS & LS AAS & CS AAS & LS AAS & CS AAS & LS AAS \\
\hline Melissa & $13,2 \pm 0,5$ & $13,9 \pm 0,2$ & $22,7 \pm 0,9$ & $22,2 \pm 0,1$ & $4,7 \pm 0,2$ & $4,3 \pm 0,3$ \\
\hline Ginkgo Biloba & $33,3 \pm 1,9$ & $32,5 \pm 2,5$ & $9,3 \pm 0,4$ & $9,0 \pm 0,1$ & $7,2 \pm 0,4$ & $6,7 \pm 0,1$ \\
\hline Hortelã & $18,4 \pm 0,6$ & $17,8 \pm 0,1$ & $22,8 \pm 0,7$ & $23,6 \pm 0,5$ & $8,4 \pm 0,3$ & $8,0 \pm 0,2$ \\
\hline Sene & $45,9 \pm 0,2$ & $46,3 \pm 0,3$ & $9,1 \pm 0,6$ & $9,7 \pm 0,1$ & $3,9 \pm 0,2$ & $3,6 \pm 0,1$ \\
\hline Cáscara Sagrada & $16,9 \pm 1,1$ & $16,1 \pm 0,7$ & $3,9 \pm 0,2$ & $3,6 \pm 0,1$ & $1,7 \pm 0,1$ & $1,6 \pm 0,1$ \\
\hline Graviola & $11,5 \pm 0,7$ & $10,8 \pm 0,1$ & $9,4 \pm 0,3$ & $9,8 \pm 0,1$ & $3,3 \pm 0,3$ & $3,5 \pm 0,2$ \\
\hline Poejo & $7,7 \pm 0,4$ & $7,3 \pm 0,1$ & $25,6 \pm 1,8$ & $26,9 \pm 2,0$ & $2,1 \pm 0,2$ & $2,0 \pm 0,1$ \\
\hline Ginseng & $2,3 \pm 0,1$ & $2,0 \pm 0,1$ & $25,0 \pm 0,8$ & $25,5 \pm 0,1$ & $1,6 \pm 0,1$ & $1,5 \pm 0,1$ \\
\hline Pata de Vaca & $20,3 \pm 0,8$ & $19,7 \pm 0,5$ & $16,1 \pm 1,0$ & $16,9 \pm 0,1$ & $2,4 \pm 0,2$ & $2,7 \pm 0,2$ \\
\hline Erva Doce & $7,3 \pm 0,4$ & $6,8 \pm 0,4$ & $18,7 \pm 1,1$ & $19,4 \pm 0,9$ & $1,8 \pm 0,1$ & $1,9 \pm 0,1$ \\
\hline Boldo do Chile & $7,9 \pm 0,3$ & $7,2 \pm 0,3$ & $13,7 \pm 0,6$ & $14,3 \pm 0,2$ & $1,0 \pm 0,1$ & $0,9 \pm 0,1$ \\
\hline Camomila & $5,5 \pm 0,2$ & $5,2 \pm 0,1$ & $27,9 \pm 1,1$ & $28,4 \pm 0,6$ & $2,3 \pm 0,2$ & $2,2 \pm 0,2$ \\
\hline Centelha Asiática & $6,6 \pm 0,3$ & $6,0 \pm 0,2$ & $25,1 \pm 1,2$ & $24,7 \pm 0,4$ & $2,2 \pm 0,2$ & $2,1 \pm 0,1$ \\
\hline Guaçatonga & $7,8 \pm 0,4$ & $8,2 \pm 0,1$ & $26,4 \pm 0,9$ & $25,6 \pm 0,9$ & $4,4 \pm 0,4$ & $4,7 \pm 0,3$ \\
\hline Chapéu de Couro & $8,5 \pm 0,3$ & $8,1 \pm 0,1$ & $38,3 \pm 1,3$ & $39,6 \pm 1,5$ & $2,5 \pm 0,2$ & $2,8 \pm 0,2$ \\
\hline Erva Cidreira & $2,7 \pm 0,2$ & $2,5 \pm 0,1$ & $25,7 \pm 0,8$ & $26,9 \pm 0,5$ & $0,43 \pm 0,06$ & $0,40 \pm 0,03$ \\
\hline Espinheira Santa & $9,2 \pm 0,5$ & $8,5 \pm 0,7$ & $10,2 \pm 0,5$ & $10,7 \pm 0,4$ & $2,4 \pm 0,2$ & $2,6 \pm 0,1$ \\
\hline Guaco & $10,7 \pm 0,9$ & $10,0 \pm 0,8$ & $25,7 \pm 0,7$ & $25,1 \pm 0,8$ & $1,1 \pm 0,1$ & $1,2 \pm 0,1$ \\
\hline Carqueja & $5,3 \pm 0,4$ & $5,9 \pm 0,1$ & $18,5 \pm 0,6$ & $18,1 \pm 0,7$ & $0,35 \pm 0,02$ & $0,32 \pm 0,02$ \\
\hline Cavalinha & $12,8 \pm 0,5$ & $12,2 \pm 0,5$ & $19,1 \pm 1,1$ & $19,5 \pm 1,4$ & $2,2 \pm 0,1$ & $2,0 \pm 0,1$ \\
\hline
\end{tabular}




\section{CONCLUSÕES}

O arranjo instrumental da técnica HR-CS FAAS permitiu monitorar de modo rápido e sequencial as linhas secundárias do Ca em 239,856 nm, do K em 404,414 nm e a linha alternativa do $\mathrm{Mg}$ em 202,588 $\mathrm{nm}$. Medir a absorbância do $\mathrm{Mg}$ na asa do comprimento de onda secundário foi essencial para estender a faixa linear de trabalho até $80 \mathrm{mg} \mathrm{L}^{-1}$ e viabilizar a determinação conjunta de $\mathrm{Ca}, \mathrm{K}$ e $\mathrm{Mg}$ em alíquotas únicas de digeridos de plantas medicinais, evitando diluições sucessivas de amostra. Os resultados foram compatíveis com os obtidos por espectrometria de absorção atômica com fonte de linhas.

\section{Agradecimentos}

Os autores agradecem a FAPESP (Proc. 09/52480-0) e FUNDUNESP (Proc. 01113/11) pelo auxílio concedido, a CAPES pela bolsa de estudos de J.F.R, e ao CNPq pela bolsa de produtividade em pesquisa de J.A.G.N.

\section{REFERÊNCIAS}

[1] Andrade, E. C. B.; Alves, S. P.; Takase, I. Ciênc. Tecnol. Aliment. 25 (2005) 844.

[2] Welz, B.; Sperling, M. Atomic Absorption Spectrometry. $3^{\text {rd }}$ ed. Weinheim: Wiley-VCH; 1999.

[3] Weng, D. Spectrosc. and Spectral Anal. 24 (2004) 1458 .

[4] Oliveira, S. R.; Gomes Neto, J. A.; Nóbrega, J. A.; Jones, B. T. Spectrochim. Acta Part B 65 (2010) 316.

[5] Oliveira, S. R.; Raposo Jr. J. L.; Gomes Neto, J. A. Spectrochim Acta Part B 64 (2009) 593.

[6] Welz, B.; Becker-Ross, H.; Florek, S.; Heitmann, U. High-Resolution Continiuum Source AAS. The Better Way to Do Atomic Absorption Spectrometry. $1^{\text {st }}$ ed. Weinheim: Wiley-VCH; 2005.

[7] Welz, B.; Becker-Ross, H.; Florek, S.; Heitmann, U.; Vale, M.G.R. J. Braz. Chem. Soc. 14 (2003) 220.

[8] Raposo Jr, J. L.; Oliveira, S. R.; Caldas, N. M.; Gomes Neto, J.A. Anal. Chim. Acta 627 (2008) 198.

[9] Krug F.J. (Ed.). Métodos de Preparo de Amostras: fundamentos sobre preparo de amostras orgânicas e inorgânicas para análise elementar. 1st ed. Piracicaba; 2008.

[10] Cardili, C.; Raposo Jr., J. L.; Cavalheiro, A. J.; Gomes Neto, J. A. Braz. J. Anal. Chem., prelo.
[11] Malavolta, E. Elementos de nutrição mineral de plantas. $1^{\text {st }}$ ed. São Paulo: Agronômica Ceres Ltda; 1980 .

[12] Currie, L. A. Anal. Chim. Acta 391 (1999) 105. 